\title{
T-Matrix Determination of Effective Permittivity for Three-Dimensional Dense Random Media
}

\author{
Paul R. Siqueira, Member, IEEE, and Kamal Sarabandi, Senior Member, IEEE
}

\begin{abstract}
In this paper, we present a full wave method for determining the effective permittivity for random media in three dimensions. The type of media addressed is composed of spherical dielectric particles in a homogeneous dielectric background. The particle volume fraction ranges from 0 to $40 \%$ and dielectric contrast may be significantly different from the background medium. The method described relies on the $T$-matrix approach for solving Maxwell's equations using a spherical wave expansion in conjunction with a Monte-Carlo simulation for calculating the mean scattered field confined within a prescribed fictitious boundary. To find the effective permittivity, the mean scattered field is compared with that of a homogeneous scatterer whose shape is defined by the fictitious boundary and its dielectric constant is varied until the scattered fields are matched. A complete description of the T-matrix approach is given along with an explanation of why the recursive form of this technique (RATMA [3]) cannot be used for addressing this problem. After the method development is completed, the results of our numerical technique are compared against the theoretical methods of the quasi-crystalline approximation and the effective field approximation to demonstrate the region of validity of the theoretical methods. The examples contained within the paper use between 30 and 120 included spheres (with radii ranging from from $k a=0.6$ to 0.8 ) within a larger, fictitious sphere of diameter $k D=8.4$.
\end{abstract}

Index Terms-Electromagnetic propagation in random media, T-matrix method.

\section{INTRODUCTION}

A fundamental macroscopic electromagnetic characteristic of any material is that of its permittivity. Permittivity is the scalar constant that relates electric flux density of the electric field and ultimately, for a large majority of media that are nonmagnetic, is the constant that describes the phase and group velocity as well as power loss of the field as it propagates through the medium. When the medium under consideration is composed of a number of discrete components, the net permittivity of the material is termed the effective permittivity. The loss of power to a propagating coherent field can take on one of two forms - that of absorption (and subsequent conversion to thermal energy) and that of scattering, whereby the coherent field is scattered into a generally radiating incoherent

Manuscript received March 27, 1998; revised September 23, 1999. This work was carried out in part at the Jet Propulsion Laboratory, California Institute of Technology, Pasadena, CA, under a contract with the National Aeronautics and Space Administration. This work was also supported by ARO Grant DAAH04-93-G-0156 and HPCC/ESS Grand Challenge Grant for furthering the application of supercomputers for SAR interferometry and imaging science.

P. R. Siqueira is with the Jet Propulsion Laboratory, Pasadena, CA 91109-8199 USA.

K. Sarabandi is with the University of Michigan, Ann Arbor, MI 48109-2122 USA.

Publisher Item Identifier S 0018-926X(00)01269-2. field. This second form of power loss is much more difficult to characterize than absorption because of the complex nature of scattering.

The simplest approach for determining effective permittivity is to ignore the effect of scattering on the mean field which illuminates the components of the medium. In the derivation of the Polder-Van Santen mixing formula [9], the mean field induces a dipole moment within the fundamental components of the medium, which in turn can be used to derive the effective permittivity. Because of the assumption regarding the mean field and ignoring the scattering, it is inherently a low-frequency approach.

When scattering is taken into account, the problem becomes considerably more complex. Scattering is an important component for determining effective permittivity when the scatterer size is on the same dimensional scale as the observing wavelength (i.e., a low-frequency estimation can no longer be applied). In this instance the interaction of the incident field with all of the scatterers must be simultaneously taken into account. Depending on the density of the medium, this multiple scattering may be theoretically truncated so that the mean field can be determined and the problem solved. Such is the case for Foldy's approximation (also called the effective field approximation), for single scattering, and the quasi-crystalline approximation, which accounts for all pair-wise scattering.

This paper addresses the problem of numerically determining the permittivity of a material that contains discrete dielectric (nontenuous) components whose dimension is on the same order of the electromagnetic field, taking into account all orders of multiple scattering. The approach described stems from an extensive study that has been accomplished for addressing the two-dimensional (2-D) version of this problem. That is, to develop a reliable and consistent numerical technique that can determine both real and imaginary components of effective permittivity which is not limited in principal to the number density of scatterers or to a high ratio of absorption to scattering losses within the system.

We begin by first reviewing the technique and results as applied to the 2-D random media problem. While not critical to the technique overall, the 2-D version utilized the method of moments to solve Maxwell's equations to determine the scattered fields. Because of the additional dimension in the three-dimensional (3-D) problem, it has been necessary to implement an alternative numerical approach to solve Maxwell's equations for the random medium. For this study we chose the T-matrix method first proposed by Waterman [18] and later refined by Chew [3]. This approach, along with its limitations, is discussed in the second section of this paper. While the recursive form of 

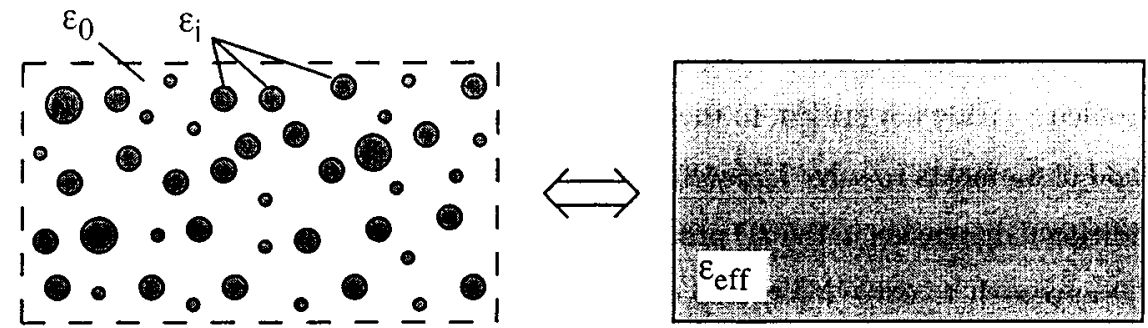

Fig. 1. Model for numerically determining $\epsilon_{\mathrm{eff}}$ for a random medium.

T-matrix technique did not prove to be appropriate for our application, we were nevertheless able to use the T-matrix method in its more traditional form. Although limiting the solution space (in terms of number density) for determining effective permittivity, we will demonstrate its application as well as provide a comparison with theoretically derived results.

\section{Two-Dimensional TeCHNIQUe FOR CHARACTERIZING $\epsilon_{\mathrm{eff}}$}

The technique described here is a numerical method for determining the effective permittivity of a random medium. This method, based upon the coherent scattered electric field from a bounded volume, is developed in contrast to an incoherent method for determining the same quantity [16]. The coherent numerical method that follows and its application have been developed by Siqueira and Sarabandi [11], [12], [14] as well as Zurk et al. [19], [20].

The concept itself is straightforward and is explained here as a prelude to the 3-D problem (for a more complete description see [13]). We begin by extracting a bounded sample of the random medium and illuminating it with a known electric field. The scattered field from this sample will be a superposition of the coherent field, resulting from the boundary and the incoherent field due to the inhomogeneities within the boundary. Averaging together the complex valued scattered fields for many samples of the medium at each observation angle will yield only the coherent field, which is directly related to the shape of the boundary and the effective permittivity of the random medium (see Fig. 1). Given the fact that we have control of the boundary size and shape, a canonical shape can be chosen whose scattering solution is known or may be numerically determined. By comparing the coherent scattered field from the random medium samples with the scattered field from the canonical shape, the permittivity of the canonical shape can be changed until the two sets of scattered fields are the same.

The process described above has been implemented and tested extensively in two dimensions as well as compared to theoretical models. For a complete comparison of the 2-D numerical technique with the theoretical models of the mixing formula, Foldy's approximation, and the quasi-crystalline approximation, the reader is referred to [14].

\section{T-MATRIX}

In two dimensions the method of moments was used to solve Maxwell's equations because of the exact nature of the solution for canonical inclusion shapes of squares and circles. In three dimensions, however, application of the method of moments is much more cumbersome due to the large number of unknowns (vector volume currents) inherent to the problem and the complexity of discretizing these currents. As a consequence, it is unrealistic to solve any 3-D large-scale problems using the method of moments. To circumvent this difficulty, the T-matrix method (first proposed by Waterman [18], Peterson and Strom [9], and later developed into a recursive technique, RATMA, by Wang and Chew [17]) was used to solve for the scattering due to a large number of scatterers. The recursive form of the T-matrix method is particularly appealing because the computational complexity scales as $n_{\max }^{7 / 3}$ rather than $n_{\max }^{3}$ of the traditional approach, where $n_{\max }$ is the total number of scatterers (this assumes that the enclosing volume scales linearly with $n_{\max }$ ). Because the development and application of the recursive T-matrix solution technique is fairly recent, it is reviewed here for clarity, completeness, and also to highlight its uses and limitations.

In summary, the T-matrix has the following advantages.

1) The spherical wave expansion of the T-matrix automatically accounts for near-field interaction.

2) The T-matrix could potentially be used iteratively to mitigate the necessity for inverting large matrices.

3) The T-matrix works best for spherically shaped particles, a good approximation for media such as sand and snow grains; more complex geometries could be approximated by building collections of spheres or by employing extended boundary conditions.

4) Depending on the dimension/frequency scale of the scattering components, the number of terms used in the spherical wave expansion can be controlled.

5) The T-matrix is exact given a sufficient number of spherical wave basis functions.

\section{A. Formulation}

The formulation of the generalized recursive aggregate T-matrix algorithm (RATMA) is based upon the spherical wave expansion of electromagnetic fields. These functions, composed of the field vectors $\bar{M}$ and $\bar{N}$ are arranged into a vector $\bar{\Psi}$ of varying spherical waveform expansions. In the first case, the vectors are 3-D, one dimension for each direction in space. For $\overline{\boldsymbol{\Psi}}$, however, the vectors have a dimension of $2 P_{\max }\left(P_{\max }+2\right)$, where the parameter $P_{\max }$ will be explained shortly. Because of the greater need to work with vectors of spherical wave functions in this context, vector field quantities (such as $\bar{M}$ and $\bar{N}$ ) normally specified by bold face type and an overbar will revert to nonemphasized characters (i.e., $M$ and $N$ ) with the excep- 
tion of positional vectors such as $\bar{r}$. Thus, a vector of spherical wave functions can be written as

$$
\overline{\boldsymbol{\Psi}}=\left[\begin{array}{lll}
\cdots M_{l m} \cdots & \cdots N_{l m} \cdots
\end{array}\right]^{t}
$$

which is a column vector (the superscript $t$ indicating a matrix transpose) and where

$$
\begin{aligned}
M_{l m} & =\nabla(\bar{r} \psi) \quad \text { and } \quad N_{l m}=\frac{1}{k_{0}} \nabla \times M \\
\psi_{l m} & =h_{l}^{(1)}\left(k_{0} r\right) Y_{l m}(\theta, \phi) \quad \text { and } \\
\operatorname{Rg} \psi_{l m} & =j_{l}\left(k_{0} r\right) Y_{l m}(\theta, \phi) .
\end{aligned}
$$

In the above equations, $j_{l}$ and $h_{l}^{(1)}$ are the spherical Bessel and Hankel functions of order $l$ and $Y_{l m}$ is the associated Legendre polynomial, defined in this paper as

$$
Y_{l m}(\theta, \phi)=(-1)^{m} \sqrt{\frac{(2 l+1)}{4 \pi} \frac{(l-m) !}{(l+m) !}} P_{l}^{m}(\cos \theta) e^{i n \phi}
$$

where $P_{l}^{m}(\cos \theta)$ are the ordinary Legendre polynomials given in [1]. It is necessary to exactly specify which form of the associated Legendre polynomial is being used because the convention varies from application to application (see [3] versus [16]). A generalized incident field $E^{\text {inc }}$ may be decomposed into spherical wave functions as in

$$
E^{\text {inc }}=\operatorname{Rg} \overline{\boldsymbol{\Psi}}^{t} \overline{\boldsymbol{a}}^{\text {inc }}
$$

where $\bar{a}^{\mathrm{inc}}$ is a column vector of incident field coefficients. For plane wave incidence, the incident field coefficients are given by

$$
\begin{aligned}
\overline{\boldsymbol{a}}^{\mathrm{inc}}= & {\left[\begin{array}{ll}
\overline{\boldsymbol{a}}^{M} & \overline{\boldsymbol{a}}^{N}
\end{array}\right]^{t} } \\
a_{p q}^{M}= & (-1)^{q} i^{p} e^{-i q \phi} \sqrt{\frac{8 \pi}{p(p+1)}} \\
& \cdot\left[\hat{\theta} i t_{p}^{-q}(\cos (\theta))-\hat{\phi} s_{p}^{-q}(\cos (\theta))\right] \\
a_{p q}^{N}= & (-1)^{q} i^{p-1} e^{-i q \phi} \sqrt{\frac{8 \pi}{p(p+1)}} \\
& \cdot\left[\hat{\theta} s_{p}^{-q}(\cos (\theta))-\hat{\phi} i t_{p}^{-q}(\cos (\theta))\right]
\end{aligned}
$$

which in itself contains vector field quantities, making the expression in (5) a dyad; the leading vector field relating to the received polarization and the trailing vector field the transmit polarization. The functions, $s$ and $t$ are related to the associated Legendre polynomial and its derivative and can be found in [14]. In the above expressions, $p$ refers to the order of the dipole moment and $q$ is the dipole number such that $q=\{-p,-p+1, \cdots, p\}$ and $p=1, \cdots, P_{\max }$, where $P_{\max }$ is the truncation number for the number of dipole moments used in the calculation. For a given $P_{\max }$, the number of elements in $\overline{\boldsymbol{a}}^{\mathrm{inc}}$ is $2 P_{\max }\left(P_{\max }+2\right)$. Similarly, the scattered electric field can be expressed in terms of the scattered field coefficients $\overline{\boldsymbol{a}}^{\text {sca }}$ such that

$$
E^{\mathrm{sca}}=\overline{\boldsymbol{\Psi}}^{t} \overline{\boldsymbol{a}}^{\mathrm{sca}} .
$$

The T-matrix (or transition matrix) is then defined by the relation

$$
\overline{\boldsymbol{a}}^{\mathrm{sca}}=\overline{\overline{\boldsymbol{T}}} \overline{\boldsymbol{a}}^{\mathrm{inc}} .
$$

For a single sphere, centered at the origin, the T-matrix reverts to the Mie series. For the more general problem, it will be necessary to sum together the effects of a number of spheres, displaced from the origin by the vector $\overline{r r}_{i}$. For a single sphere, this problem can be easily addressed using the vector translation theorem as in

$$
E^{\text {sca }}=\overline{\boldsymbol{\Psi}}^{t} \overline{\overline{\boldsymbol{\beta}}}_{01}\left(\overline{r r}_{1}\right) \cdot \overline{\overline{\boldsymbol{T}}} \cdot \overline{\overline{\boldsymbol{\beta}}}_{10}\left(\bar{r}_{1} \bar{r}\right) \overline{\boldsymbol{a}}^{\mathrm{inc}} .
$$

In the above equation, the vector translation matrix $\overline{\overline{\boldsymbol{\beta}}}_{i j}$ indicates a translation of spherical wave functions centered around the $i$ th coordinate system to the $j$ th coordinate system.

In (9) it can be seen that the incident field vector in global coordinates is transformed to the local coordinates of the scatterer, multiplied by the T-matrix, transformed back to the global coordinate system, and finally multiplied by the spherical wave basis functions in global coordinates. Use of the vector translation theorem is dependent on the point of observation with respect to the vector that describes the translation of coordinates (this will be discussed shortly).

The fundamental equation for the direct T-matrix algorithm relies on the continued application of this transformation via the application of the different forms of the vector addition theorem. Fig. 2 illustrates the multiple scattering equation given by [9] [14]

$$
\overline{\boldsymbol{w}}^{(j)}=\sum_{\substack{i=1 \\ i \neq j}}^{n_{\max }} \overline{\overline{\boldsymbol{\alpha}}}_{j i} \cdot \overline{\overline{\boldsymbol{T}}}_{i(1)} \cdot \overline{\boldsymbol{w}}^{(i)}+\overline{\overline{\boldsymbol{\beta}}}_{j 0} \cdot \overline{\boldsymbol{a}}^{\mathrm{inc}} .
$$

In (10), $\bar{w}^{(j)}$ is the vector of exciting field coefficients which describe the field on the surface $s$ encompassing the $j$ th particle and $\overline{\overline{\boldsymbol{\alpha}}}$ is a vector translation matrix similar to $\overline{\overline{\boldsymbol{\beta}}}$ with the exception that spherical Hankel functions are used instead of spherical Bessel functions. In (10), $\overline{\bar{T}}_{i(1)}$ refers to the single scattering matrix of the $i$ th particle in the absence of other particles. The fields on this surface are the sum of the scattered fields from the remaining $n_{\max }-1$ particles plus the incident field. The exciting field coefficients, $\overline{\boldsymbol{w}}^{(j)}$ are unknown and must be solved for by the matrix equation implied by (10). Once the exciting fields are known for each particle, the scattered field can be determined by summing up all of the contributions from all of the particles together.

The mathematical rules for the application of the vector addition theorem for spherical waves are often not well illustrated in the literature. In reference to Fig. 2, for an observation point on the surface $s$ and the translation of spherical wave functions centered on the $i$ th coordinate system to the $j$ th coordinate system, the vector translation theorem is given by

$$
\begin{aligned}
\overline{\boldsymbol{\Psi}}\left(k \bar{r}_{s i}\right) & =\operatorname{Rg} \bar{\Psi}\left(k \bar{r}_{s j}\right) \cdot \overline{\bar{\alpha}}_{j i} & \left|\bar{r}_{s j}\right|<\left|\bar{r}_{j i}\right| \\
\overline{\boldsymbol{\Psi}}\left(k \bar{r}_{s i}\right) & =\overline{\boldsymbol{\Psi}}\left(k \bar{r}_{s j}\right) \cdot \overline{\overline{\boldsymbol{\beta}}}_{j i} & \left|\bar{r}_{s j}\right|>\left|\bar{r}_{j i}\right| \\
\operatorname{Rg} \overline{\boldsymbol{\Psi}}\left(k \bar{r}_{s i}\right) & =\operatorname{Rg} \overline{\boldsymbol{\Psi}}\left(k \bar{r}_{s j}\right) \cdot \overline{\overline{\boldsymbol{\beta}}}_{j i} & \forall\left|\bar{r}_{s j}\right| .
\end{aligned}
$$

The incident field in (10) is expanded in terms of regular spherical wave functions, $\operatorname{Rg} \overline{\boldsymbol{\Psi}}$ centered at the global origin. Thus, (13) is used in (10) to translate these functions to a coordinate system centered at the $j$ th particle. Similarly, the product of $\overline{\overline{\boldsymbol{T}}}_{i(1)} \cdot \overline{\boldsymbol{w}}^{(i)}$ represents outgoing spherical waves $\overline{\boldsymbol{\Psi}}$, whose origin is the center of the $i$ th particle and, in turn, (11) is used to translate the spherical wave functions to a coordinate system whose 


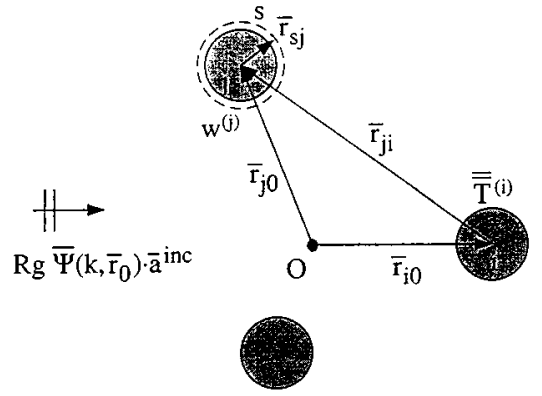

Fig. 2. Illustration of spherical wave vector translation in the multiple scattering T-matrix equation.

origin is the center of the $j$ th particle. Note that for nontouching spheres, the observation point $\bar{r}_{s j}$ will always be less than the distance between the origins of the two coordinate systems $\bar{r}_{j i}$. To develop the concept of a recursive algorithm, the T-matrix of a collection of spheres can be written in terms of the T-matrices of the individual components. This new T-matrix is often referred to as the aggregate T-matrix, designated as $\overline{\bar{\tau}}$. Extensive work on the recursive technique for determining this quantity has been performed at the University of Illinois [3]-[5], [16].

The theory behind the recursive algorithm is as follows (see Fig. 3). We begin by assuming that the aggregate scattering matrix from $n$ spheres $\overline{\overline{\boldsymbol{\tau}}}_{(n)}$ has been determined (at the beginning of the recursion, when $n=0$, the aggregate T-matrix $\overline{\bar{\tau}}_{(n)}=0$ ). The aggregate T-matrix of these $n$ spheres in the presence of an additional $n^{\prime}$ spheres can be written as

$$
\overline{\overline{\boldsymbol{\tau}}}_{n\left(n+n^{\prime}\right)}=\overline{\overline{\boldsymbol{\tau}}}_{(n)}+\overline{\overline{\boldsymbol{\tau}}}_{(n)} \cdot \sum_{j=n+1}^{n+n^{\prime}} \overline{\overline{\boldsymbol{\alpha}}}_{0 j} \cdot \overline{\overline{\boldsymbol{T}}}_{j\left(n+n^{\prime}\right)} \cdot \overline{\overline{\boldsymbol{\beta}}}_{j 0}
$$

where $\overline{\boldsymbol{T}}_{j\left(n+n^{\prime}\right)}$ is the T-matrix for the $j$ th particle in the presence of $n+n^{\prime}$ particles (including the $j$ th particle itself), $\overline{\overline{\boldsymbol{\alpha}}}$ and $\overline{\overline{\boldsymbol{\beta}}}$ are vector translation matrices as described above. Fig. 3(a) illustrates the different component terms of (14). Similarly, the T-matrix of the $j$ th sphere referenced to the origin may be written as

$$
\begin{aligned}
\overline{\overline{\boldsymbol{T}}}_{j\left(n+n^{\prime}\right)} \cdot \overline{\overline{\boldsymbol{\beta}}}_{j 0}=\overline{\overline{\boldsymbol{T}}}_{j(1)} \cdot \overline{\overline{\boldsymbol{\beta}}}_{j 0}+\overline{\overline{\boldsymbol{T}}}_{j(1)} \cdot \overline{\overline{\boldsymbol{\alpha}}}_{j 0} \cdot \overline{\overline{\boldsymbol{\tau}}}_{n\left(n+n^{\prime}\right)}+\overline{\overline{\boldsymbol{T}}}_{j(1)} \\
\cdot \sum_{\substack{i=n+1 \\
i \neq j}}^{n+n^{\prime}} \overline{\overline{\boldsymbol{\alpha}}}_{j i} \cdot \overline{\overline{\boldsymbol{T}}}_{i\left(n+n^{\prime}\right)} \cdot \overline{\overline{\boldsymbol{\beta}}}_{i 0} .
\end{aligned}
$$

The components of (15) are illustrated in Fig. 3(b). In (14) and (15) the principle unknown is the scattering matrix of the $j$ th particle in the presence of $n+n^{\prime}$ scatterers (i.e., $\overline{\bar{T}}_{j\left(n+n^{\prime}\right)} \overline{\bar{\beta}}_{j 0}$ ). By substituting (14) into (15), an algebraic expression can be written for these unknowns

$$
\begin{aligned}
& \sum_{i=n+1}^{n+n^{\prime}} \overline{\overline{\boldsymbol{\beta}}}_{A_{j i}} \cdot \overline{\overline{\boldsymbol{T}}}_{i\left(n+n^{\prime}\right)} \cdot \overline{\overline{\boldsymbol{\beta}}}_{i 0} \\
& =-\overline{\boldsymbol{T}}_{j(1)} \cdot\left[\overline{\overline{\boldsymbol{\beta}}}_{j 0}+\overline{\overline{\boldsymbol{\alpha}}}_{j 0} \cdot \overline{\overline{\boldsymbol{\tau}}}_{(n)}\right], \\
& j=n+1, \cdots, n+n^{\prime}
\end{aligned}
$$

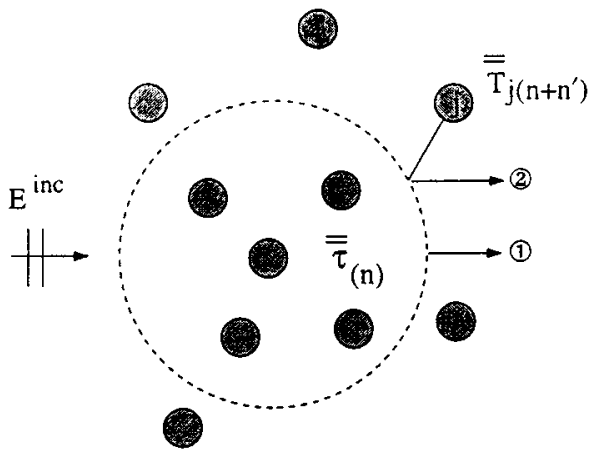

(a)

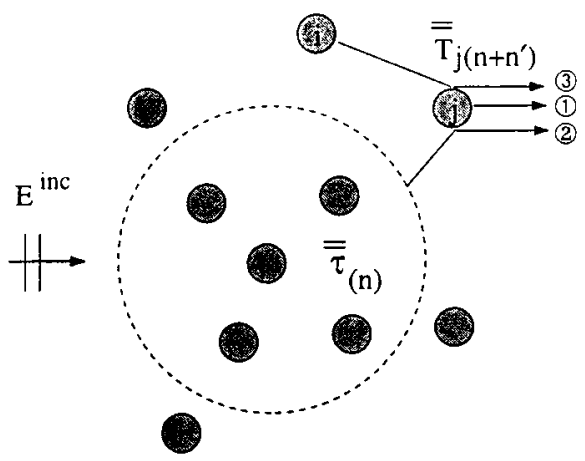

(b)

Fig. 3. (a) Scattering from $n$ spheres in the presence of $n+n^{\prime}$ spheres. (b) Scattering from sphere $j$ in the presence of $n+n^{\prime}$ spheres.

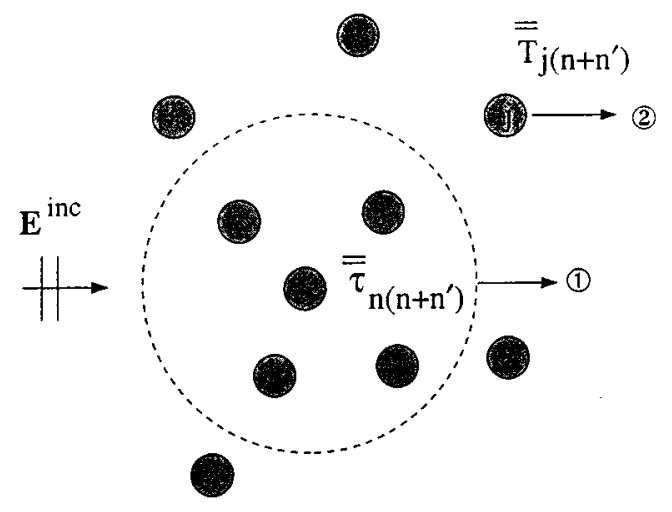

Fig. 4. Illustration of (18).

where

$$
\overline{\overline{\boldsymbol{\beta}}} A_{j i}=\left\{\begin{array}{ll}
-\left[\overline{\overline{\boldsymbol{\beta}}} I-\overline{\overline{\boldsymbol{T}}}_{j(1)} \cdot \overline{\overline{\boldsymbol{\alpha}}}_{j 0} \cdot \overline{\overline{\boldsymbol{\tau}}}_{(n)} \cdot \overline{\overline{\boldsymbol{\alpha}}}_{0 j}\right] & i=j \\
\overline{\overline{\boldsymbol{T}}}_{j(1)} \cdot \overline{\overline{\boldsymbol{\alpha}}}_{j 0} \cdot \overline{\overline{\boldsymbol{\tau}}}_{(n)} \cdot \overline{\overline{\boldsymbol{\alpha}}}_{0 j}+\overline{\overline{\boldsymbol{T}}}_{j(1)} \cdot \overline{\overline{\boldsymbol{\alpha}}}_{j i} & i \neq j
\end{array} .\right.
$$

Given a solution for $\overline{\overline{\boldsymbol{T}}}_{j\left(n+n^{\prime}\right)} \cdot \overline{\overline{\boldsymbol{\beta}}}_{j 0}$, the aggregate T-matrix of $n+n^{\prime}$ scatterers is written as (see Fig. 4)

$$
\overline{\overline{\boldsymbol{\tau}}}_{\left(n+n^{\prime}\right)}=\overline{\overline{\boldsymbol{\tau}}}_{n\left(n+n^{\prime}\right)}+\sum_{j=n+1}^{n+n^{\prime}} \overline{\overline{\boldsymbol{\beta}}}_{0 j} \cdot \overline{\overline{\boldsymbol{T}}}_{j\left(n+n^{\prime}\right)} \cdot \overline{\overline{\boldsymbol{\beta}}}_{j 0} .
$$


After substituting (14) into (18), an expression can be written for the aggregate T-matrix in terms of known quantities, $\overline{\bar{\tau}}_{(n)}$ and $\overline{\overline{\boldsymbol{T}}}_{j\left(n+n^{\prime}\right)} \cdot \overline{\overline{\boldsymbol{\beta}}}_{j 0}$

$\overline{\overline{\boldsymbol{\tau}}}_{\left(n+n^{\prime}\right)}=\overline{\bar{\tau}}_{(n)}+\sum_{j=n+1}^{n+n^{\prime}}\left(\overline{\overline{\boldsymbol{\beta}}}_{0 j}+\overline{\bar{\tau}}_{(n)} \cdot \overline{\overline{\boldsymbol{\alpha}}}_{0 j}\right) \cdot \overline{\overline{\boldsymbol{T}}}_{j\left(n+n^{\prime}\right)} \cdot \overline{\overline{\boldsymbol{\beta}}}_{j 0}$.

The critical components of the recursive algorithm are (16), (17), and (19). The first of these, (16) may be written as a matrix of matrices, as in

$$
\begin{gathered}
{\left[\begin{array}{cccc}
\overline{\overline{\boldsymbol{A}}}_{n+1, n+1} & \overline{\overline{\boldsymbol{A}}}_{n+1, n+2} & \cdots & \overline{\overline{\boldsymbol{A}}}_{n+1, n+n^{\prime}} \\
\overline{\overline{\boldsymbol{A}}}_{n+2, n+1} & \overline{\overline{\boldsymbol{A}}}_{n+2, n+2} & \cdots & \overline{\overline{\boldsymbol{A}}}_{n+2, n+n^{\prime}} \\
\vdots & \vdots & \ddots & \vdots \\
\overline{\bar{A}}_{n+n^{\prime}, n+1} & \overline{\overline{\boldsymbol{A}}}_{n+n^{\prime}, n+2} & \cdots & \overline{\overline{\boldsymbol{A}}}_{n+n^{\prime}, n+n^{\prime}}
\end{array}\right]} \\
\cdot\left[\begin{array}{c}
\overline{\overline{\boldsymbol{T}}}_{n+1,\left(n+n^{\prime}\right)} \cdot \overline{\overline{\boldsymbol{\beta}}}_{n+1,0} \\
\overline{\overline{\boldsymbol{T}}}_{n+2,\left(n+n^{\prime}\right)} \cdot \overline{\overline{\boldsymbol{\beta}}}_{n+2,0} \\
\vdots \\
\overline{\overline{\boldsymbol{T}}}_{n+n^{\prime},\left(n+n^{\prime}\right)} \cdot \overline{\overline{\boldsymbol{\beta}}}_{n+n^{\prime}, 0}
\end{array}\right] \\
=\left[\begin{array}{c}
-\overline{\overline{\boldsymbol{T}}}_{n+1(1)}\left(\overline{\overline{\boldsymbol{\beta}}}_{n+1,0}+\overline{\overline{\boldsymbol{\alpha}}}_{n+1,0} \cdot \overline{\overline{\boldsymbol{\tau}}}_{(n)}\right) \\
-\overline{\overline{\boldsymbol{T}}}_{n+2(1)}\left(\overline{\overline{\boldsymbol{\beta}}}_{n+2,0}+\overline{\overline{\boldsymbol{\alpha}}}_{n+2,0} \cdot \overline{\overline{\boldsymbol{\tau}}}_{(n)}\right) \\
\vdots \\
\overline{\overline{\boldsymbol{T}}}_{n+n^{\prime}(1)}\left(\overline{\overline{\boldsymbol{\beta}}}_{n+n^{\prime}, 0}+\overline{\overline{\boldsymbol{\alpha}}}_{n+n^{\prime}, 0} \cdot \overline{\overline{\boldsymbol{\tau}}}_{(n)}\right)
\end{array}\right]
\end{gathered}
$$

which can be more concisely written as

$$
\overline{\overline{\mathcal{A}}} \overline{\overline{\mathcal{C}}}=\overline{\overline{\mathcal{B}}}
$$

Furthermore, (19) can be written in matrix form as

$$
\begin{aligned}
\overline{\bar{\tau}}_{\left(n+n^{\prime}\right)}=\overline{\overline{\boldsymbol{\tau}}}_{(n)}+\left[\left(\overline{\overline{\boldsymbol{\beta}}}_{0, n+1}+\overline{\overline{\boldsymbol{\tau}}}_{(n)} \cdot \overline{\overline{\boldsymbol{\alpha}}}_{0, n+1}\right)\right. \\
\left.\cdot\left(\overline{\overline{\boldsymbol{\beta}}}_{0, n+n^{\prime}}+\overline{\overline{\boldsymbol{\tau}}}_{(n)} \cdot \overline{\overline{\boldsymbol{\alpha}}}_{0, n+n^{\prime}}\right)\right] \\
\\
\cdot\left[\begin{array}{c}
-\overline{\overline{\boldsymbol{T}}}_{n+1(1)}\left(\overline{\overline{\boldsymbol{\beta}}}_{n+1,0}+\overline{\overline{\boldsymbol{\alpha}}}_{n+1,0} \cdot \overline{\overline{\boldsymbol{\tau}}}_{(n)}\right) \\
\vdots \\
-\overline{\overline{\boldsymbol{T}}}_{n+n^{\prime}(1)}\left(\overline{\overline{\boldsymbol{\beta}}}_{n+n^{\prime}, 0}+\overline{\overline{\boldsymbol{\alpha}}}_{n+n^{\prime}, 0} \cdot \overline{\overline{\boldsymbol{\tau}}}_{(n)}\right)
\end{array}\right]
\end{aligned}
$$

or

$$
\overline{\overline{\boldsymbol{\tau}}}_{\left(n+n^{\prime}\right)}=\overline{\overline{\boldsymbol{\tau}}}_{(n)}+\overline{\overline{\mathcal{D}}} \cdot \overline{\overline{\mathcal{C}}}
$$

From the above, (21) can be used to solve for $\overline{\overline{\mathcal{C}}}$ which can then be substituted into (23) to determine the aggregate T-matrix.

The recursive form of the T-matrix just derived is a superset of direct T-matrix method described in [9], [14], [17]. If at the beginning of the algorithm $n^{\prime}=n_{\max }$, the total number of spheres to be added (16), (18), and (19) reduce to a single matrix inversion, which is equivalent to the direct T-matrix method. The equivalence between the multiple scattering equation for the direct T-matrix described by (10) and the T-matrix algorithm developed in this paper, given by (15), can be seen by right multiplying (15) by $\overline{\boldsymbol{a}}^{\text {inc }}$ and making the substitution

$$
\overline{\overline{\boldsymbol{T}}}_{j(1)} \cdot \overline{\boldsymbol{w}}^{(j)}=\overline{\overline{\boldsymbol{T}}}_{j(N)} \cdot \overline{\overline{\boldsymbol{\beta}}}_{j 0} \cdot \overline{\boldsymbol{a}}^{\mathrm{inc}}
$$

which would make the initial starting point of the two methods identical.
TABLE I

MATRIX DimENSIONS FOR THE RECURSIVE T-MATRIX

\begin{tabular}{r|c|c|c|c|c|c|c|c|c|c}
\hline variable: & $\overline{\overline{\boldsymbol{A}}}_{j i}$ & $\overline{\overline{\boldsymbol{T}}}_{j(n)}$ & $\overline{\overline{\boldsymbol{\beta}}}_{j i}, \overline{\overline{\bar{\alpha}}}_{j i}$ & $\overline{\overline{\boldsymbol{\beta}}}_{j 0}, \overline{\overline{\boldsymbol{\alpha}}}_{j 0}$ & $\overline{\overline{\boldsymbol{\beta}}}_{0 j}, \overline{\bar{\alpha}}_{0 j}$ & $\overline{\overline{\boldsymbol{\tau}}}_{(n)}$ & $\overline{\overline{\mathcal{A}}}$ & $\overline{\overline{\mathcal{B}}}$ & $\overline{\overline{\mathcal{C}}}$ & $\overline{\overline{\mathcal{D}}}$ \\
\hline rows: & $K$ & $K$ & $K$ & $K$ & $P$ & $P$ & $n^{\prime} K$ & $n^{\prime} K$ & $n^{\prime} K$ & $P$ \\
\hline cols: & $K$ & $K$ & $K$ & $P$ & $K$ & $P$ & $n^{\prime} K$ & $P$ & $P$ & $n^{\prime} K$ \\
\hline
\end{tabular}

The dimension of the arrays utilized in the recursive T-matrix method is an important parameter. Array dimensions are governed by the order of the dipole moment used in calculating the T-matrices of individual scatterers, denoted as $K_{\max }$, the order of the dipole moment used for the aggregate T-matrix, denoted as $P_{\max }$, and the number of new scatterers added at each iteration $n^{\prime}$. For a given value of $K_{\max }$ (or $P_{\max }$ ), there are a total number of $K=2 K_{\max }\left(K_{\max }+2\right)$ (or $P=2 P_{\max }\left(P_{\max }+2\right)$ ) components of the electric field (the factor of two coming from a combination of $M$ and $N$ vector fields). Table I lists the dimensions of the matrices utilized by the recursive T-matrix method in terms of these components (see Table I). Note that the one matrix inversion that must be performed for each iteration, is on $\overline{\overline{\mathcal{A}}}$, which has dimensions of $n^{\prime} K \times n^{\prime} K$. For Rayleigh sized spheres $K_{\max }=1$ and, therefore, a $6 n^{\prime} \times 6 n^{\prime}$ matrix must be inverted. Values of $n^{\prime}$ on the order of 30 spheres per iteration will maintain the matrix inversion within reasonable limits, but this will be at the sacrifice of some accuracy (which will be demonstrated in the next section).

The number of dipole moments required for the aggregate T-matrix is best determined by the formula given by Bohren and Huffman [2] as

$$
P_{\max }=k_{0} A+4\left(k_{0} A\right)^{1 / 3}+2
$$

where $k_{0}$ is the free-space wave number and $A$ is the radius of the sphere enclosing the total of $n_{\max }$ particles comprising the medium under analysis.

\section{B. Limitations of the Recursive T-Matrix Algorithm}

The T-matrix algorithm and RATMA are numerically exact in that if a sufficient number of terms of the spherical wave expansion are retained and, if the machine precision is sufficient, an exact solution to Maxwell's equations will result. Computational limits, however, require a more practical solution to these equations and limits must be put on $P_{\max }$ and $K_{\max }$ as was alluded to above.

Experience has shown that the direct T-matrix method converges to a unique solution by implementing (25) from above for the value of $P_{\max }$ and $K_{\max }=1$ to 3 for the component spheres (assuming they are less than one wavelength). Reducing $P_{\max }$ below the value specified in (25) will have the effect of giving erroneous results, while using an insufficient $K_{\max }$ will constitute a loss of power in the system (i.e., some energy is lost in the higher order terms of the spherical wave expansion).

The recursive T-matrix approach (RATMA), however, has another set of limitations with respect to the choice of $P_{\max }$. This limitation is not governed by (25) and thus requires special consideration. This limitation is best illustrated by comparing (16) and (17) derived using the traditional and recursive T-matrix algorithms for the two-sphere problem. Using these equations, it can be shown that total scattered field from the second (outer) 
sphere, calculated using the direct T-matrix approach, can be expressed by

$$
\begin{array}{r}
\overline{\overline{\boldsymbol{T}}}_{2(2)} \cdot \overline{\overline{\boldsymbol{\beta}}}_{20}=\overline{\overline{\boldsymbol{T}}}_{2(1)} \cdot\left[\overline{\overline{\boldsymbol{\alpha}}}_{21} \cdot \overline{\overline{\boldsymbol{T}}}_{1(1)} \cdot \overline{\overline{\boldsymbol{\alpha}}}_{12} \cdot \overline{\overline{\boldsymbol{T}}}_{2(2)} \overline{\overline{\boldsymbol{\beta}}}_{20}\right. \\
\left.+\overline{\overline{\boldsymbol{\alpha}}}_{21} \cdot \overline{\overline{\boldsymbol{T}}}_{1(1)} \cdot \overline{\overline{\boldsymbol{\beta}}}_{10}+\overline{\overline{\boldsymbol{\beta}}}_{20}\right] .
\end{array}
$$

Similarly, for the same quantity, the recursive algorithm gives

$$
\begin{aligned}
\overline{\overline{\boldsymbol{T}}}_{2(2)} \cdot \overline{\overline{\boldsymbol{\beta}}}_{20}=\overline{\overline{\boldsymbol{T}}}_{2(1)} \cdot\left[\overline{\overline{\boldsymbol{\alpha}}}_{20} \cdot \overline{\overline{\boldsymbol{\beta}}}_{01} \cdot \overline{\overline{\boldsymbol{T}}}_{1(1)} \cdot \overline{\overline{\boldsymbol{\beta}}}_{10} \cdot \overline{\overline{\boldsymbol{\alpha}}}_{02}\right. \\
\left.\cdot \overline{\overline{\boldsymbol{T}}}_{2(2)} \overline{\overline{\boldsymbol{\beta}}}_{20}+\overline{\overline{\boldsymbol{\alpha}}}_{21} \cdot \overline{\overline{\boldsymbol{T}}}_{1(1)} \cdot \overline{\overline{\boldsymbol{\beta}}}_{10}+\overline{\overline{\boldsymbol{\beta}}}_{20}\right] .
\end{aligned}
$$

The difference between these two equations lies in the vector translation relationships given by

$$
\begin{aligned}
& \overline{\overline{\boldsymbol{\alpha}}}_{21}=\overline{\overline{\boldsymbol{\alpha}}}_{20} \cdot \overline{\overline{\boldsymbol{\beta}}}_{01} \\
& \overline{\overline{\boldsymbol{\alpha}}}_{12}=\overline{\overline{\boldsymbol{\beta}}}_{10} \cdot \overline{\overline{\boldsymbol{\alpha}}}_{02}
\end{aligned}
$$

in matrix notation or

$$
\begin{aligned}
A_{\mu \nu, n m}\left(k \bar{r}_{12}\right)= & \sum_{i=1}^{\infty} \sum_{j=-i}^{i} A_{\mu \nu, i j}\left(k \bar{r}_{20}\right) \operatorname{Rg} A_{i j, n m}\left(k \bar{r}_{01}\right) \\
& +B_{\mu \nu, i j}\left(k \bar{r}_{20}\right) \operatorname{Rg} B_{i j, n m}\left(k \bar{r}_{01}\right) \\
B_{\mu \nu, n m}\left(k \bar{r}_{12}\right)= & \sum_{i=1}^{\infty} \sum_{j=-i}^{i} B_{\mu \nu, i j}\left(k \bar{r}_{20}\right) \operatorname{Rg} A_{i j, n m}\left(k \bar{r}_{01}\right) \\
& +A_{\mu \nu, i j}\left(k \bar{r}_{20}\right) \operatorname{Rg} B_{i j, n m}\left(k \bar{r}_{01}\right)
\end{aligned}
$$

in summation notation. In the above $\left|\bar{r}_{10}\right|<\left|\bar{r}_{20}\right|$ where (30) and (31) are the expanded form of (28) in terms of the vector addition theorem coefficients given by [3]. This form of the addition theorem is shown explicitly in summation form here to highlight the fact that the summation is truncated after $P_{\max }$ terms instead of the infinite bounds given in (30) and (31).

Returning to matrix notation of (28) and (29), we see that the right-hand side performs the operation of transferring vector spherical wave functions centered upon one of the spheres to the origin and then translating to the remaining sphere. Given an infinite number of spherical harmonics (i.e. $P_{\max }=\infty$ ) this would be equivalent to directly transforming the vector spherical wave functions from one sphere center to the other (i.e., the left-hand side of (28)). When $P_{\max }$ is finite, however, the relations given by (28) through (31) are approximate and the convergence of this relation with respect to $P_{\max }$ is a function of both the distance of the spheres from the origin and the proximity of the spheres to one another. This is due to the singular nature of Hankel functions at or near the origin.

The effect of this limitation can be observed by calculating the diagonal elements of $\overline{\overline{\boldsymbol{\alpha}}}_{21}$ directly and comparing them with the matrix product $\overline{\boldsymbol{\alpha}}_{20} \cdot \overline{\overline{\boldsymbol{\beta}}}_{01}$ as in (28). This is done in Fig. 5 for three different values of $\bar{r}_{21}(\lambda / 10,2 \lambda / 10$, and $3 \lambda / 10)$ and three different limits for the number of dipole moments used in the spherical wave expansion $\left(P_{\max }, P_{\max }+2, P_{\max }+4\right)$, where the reference $P_{\max }$ is determined by (25) and is related to the magnitude of $\bar{r}_{20}$, which is fixed at $\lambda / 2$ in this example. As $\left|\bar{r}_{21}\right|$ gets smaller, the imaginary component of $\overline{\boldsymbol{\alpha}}_{21}$ increases due to the singularity of Hankel functions when the argument is nearly zero. This is shown for the first three dipole moments in

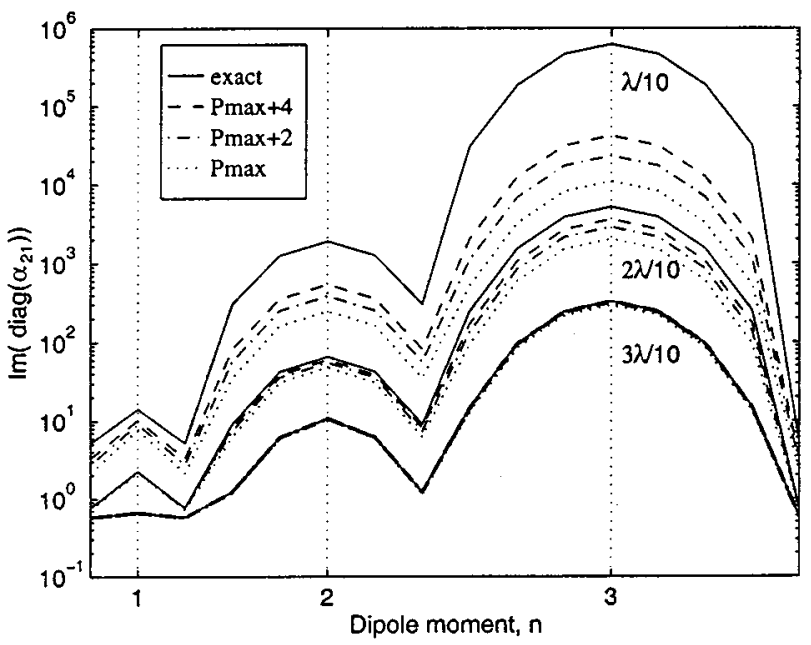

(a)

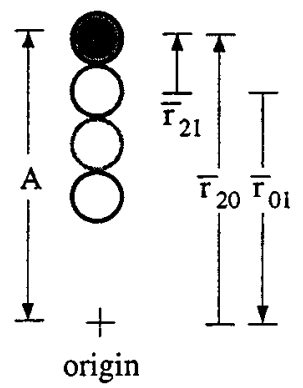

(b)

Fig. 5. Limitations of RATMA. (a) Imaginary component of the diagonal elements of $\overline{\boldsymbol{\alpha}}_{21}$ computed using the matrix product $\overline{\overline{\boldsymbol{\alpha}}}_{20} \cdot \overline{\overline{\boldsymbol{\beta}}}_{01}$ as in (28) for three different values of $\left|\bar{r}_{21}\right|$. For each $\left|\bar{r}_{21}\right|(\lambda / 10,2 \lambda / 10$, and $3 \lambda / 10)$, the exact value of $\overline{\overline{\boldsymbol{\alpha}}}_{21}(-)$ is given alongside the approximate values obtained by the matrix product with different values for the maximum number of dipole moments used (see legend). $P_{\max }$ is given by (25). (b) Physical geometry used in the analysis. Shown are the three different particle positions (open circles) in relation to the fixed particle (shaded circle) and the distance $A$ for calculating $P_{\max }$. Particle diameter is $\lambda / 10$ and $A=\lambda / 2$.

Fig. 5 for the three different distances of $\bar{r}_{21}$. As the distance $\bar{r}_{21}$ decreases, more terms in the global coordinate dipole expansion are required to reconstruct the large imaginary component of $\overline{\overline{\boldsymbol{\alpha}}}_{21}$ from the matrix multiplication between $\overline{\bar{\alpha}}_{20}$ and $\overline{\bar{\beta}}_{01}$. When the value of $\bar{r}_{21}$ is relatively large $(3 \lambda / 10)$, there are a sufficient number of terms in the global dipole expansion to reconstruct $\overline{\overline{\boldsymbol{\alpha}}}_{21}$. This can be seen in the lower set of three plots of Fig. 5. As the scattering centers get closer, however, and $\bar{r}_{21}$ gets smaller, the imaginary component of $\overline{\bar{\alpha}}_{21}$ increases and a larger number of dipole moments in the global expansion are required to maintain accuracy of the dipole expansion. Thus, for Rayleigh-sized scatterers and smaller-sized scatterers spaced closely together, the recursive T-matrix algorithm requires an unrealistically large number of dipole moments to accurately account for the interaction between scatterer centers.

The consequence of this limitation is that the accuracy of the recursive form of the T-matrix algorithm in accounting for strong interactions between neighboring spheres depends not only upon the value of $P_{\max }$ specified in (25), but also on how closely together the spheres are packed together and how much they are expected to interact. For the two-sphere problem, this error can be on the order of a 1-dB uniform loss (i.e., offset of 
power over all observation angles, as will be shown). For multiple spheres or random media the error is less predictable, but overall it should be noted that the error reduces with increasing $P_{\max }$ and decreasing electromagnetic interaction between particles. Thus, the computational advantage that the recursive version of the T-matrix method promises comes at the expense of solution accuracy.

\section{Two Interacting Sphere Example}

The following section utilizes the recursive aggregate T-matrix (RATMA) described in the previous section to demonstrate the limitation of RATMA in recursively determining the scattered field from two strongly interacting spheres. The electric field quantities that will be shown are elements of the scattering matrix $S_{r t}$ in the far field such that

$$
\frac{e^{i k_{0} r}}{k_{0} r} S_{r t}=\hat{p}_{r} \cdot E^{\mathrm{sca}} \cdot \hat{p}_{t}
$$

where $\hat{p}_{r}$ and $\hat{p}_{t}$ from above refer to the transmit and receive polarizations. The far-field components of the electric field $M$ and $N$ comprising the spherical basis functions $\overline{\boldsymbol{\Psi}}$ in the far-field region are approximated by

$$
\begin{aligned}
& M_{p q}^{F F}=i^{-p} e^{i q \phi} \frac{e^{i k_{0} r}}{k_{0} r} \frac{\left[\hat{\theta} t_{p}^{q}(\cos \theta)+\hat{\phi} i s_{p}^{q}(\cos \theta)\right]}{\sqrt{2 \pi}} \\
& N_{p q}^{F F}=i^{-p} e^{i q \phi} \frac{e^{i k_{0} r}}{k_{0} r} \frac{\left[\hat{\theta} s_{p}^{q}(\cos \theta)+\hat{\phi} i t_{p}^{q}(\cos \theta)\right]}{\sqrt{2 \pi}} .
\end{aligned}
$$

Two scenarios are considered for a pair of spheres aligned along the $z$-axis (see Fig. 6). In the first scenario, the spheres are adjacent to one another so that their interaction is maximal. In the second scenario, the position of one of the spheres is changed so that its radial distance from the origin remains the same, but it is now on the opposite side of the $z=0$ plane. This setup guarantees that the magnitude of the translation formulas used in both scenarios is the same and, therefore, the accuracy of the translation formulas will be the same between the two examples (the only difference between the examples is the degree of interaction between the two spheres). The solution for each configuration is performed using the recursive algorithm and the direct T-matrix inversion techniques. The radii of the spheres are $k a=0.63$ and the distance of the outer sphere to the origin is $k d_{30}=6.3$ (thus, $k d_{31}=1.3, k d_{32}=11.3$, and $P_{\max }=16$; the value of $P_{\max }$ was calculated by (25)).

As shown in the lower plot of Fig. 7, the two noninteracting sphere simulation gives equivalent results for both the recursive and traditional T-matrix approaches. These results have also been shown to agree with theoretical results for noninteracting spheres. When the interaction between spheres becomes significant however, we see a shift between the result obtained from the direct and recursive T-matrix algorithms. Furthermore, the recursive method more closely approximates the result given by the traditional method as the value of $P_{\max }$ is increased even though the minimal value of $P_{\max }$ specified by (25) has been surpassed. A form of this limitation is mentioned by [6] as being due to a violation of the conditions for the vector addition theorem [specified in (11) through (13)]. A violation of the vector addition theorem occurs when a source is located within the

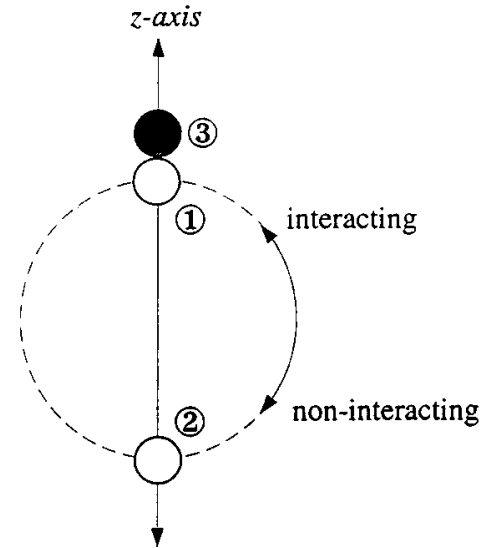

Fig. 6. Two spheres aligned along the $z$-axis. The position of the inner sphere may be adjacent to the outer sphere, or opposite of it (as shown). In the example that follows, the angle of observation is in the $z=0$ plane as measured from the incident field whose direction of propagation is defined by the $x$-axis.
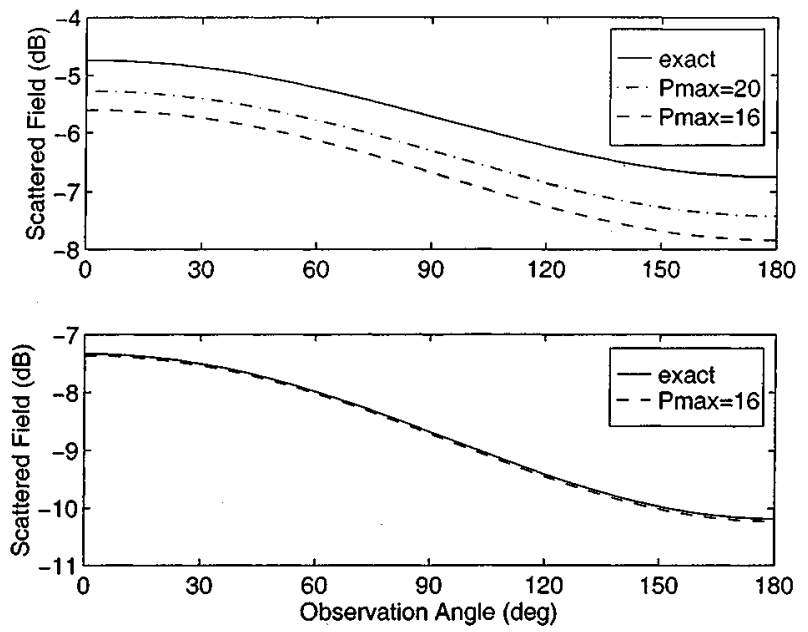

Fig. 7. Scattered field (vv-pol) from two offset spheres centered along the $z$-axis. Both plots show results for both the traditional T-matrix approach (labeled "exact") and the recursive algorithm, where $P_{\max }$ is given.

boundary of the aggregated T-matrix. The geometry used in this study precludes the possibility of this error being due to a violation of the vector addition theorem and illustrates the impact of the approximation described by (28) through (31).

The results shown by the two-sphere example illustrated by Fig. 7 indicates a loss of energy in the system when using the recursive T-matrix algorithm. This power loss can be directly be attributed to truncation of the slowly convergent infinite series expansion implied by (30) and (31).

\section{THREE-DIMENSIONAL TECHNIQUE FOR CHARACTERIZING $\epsilon_{\mathrm{eff}}$}

The 3-D technique parallels very closely the 2-D version already discussed. In this treatment, a fictitious spherical boundary is used for its ease of implementation and the ability to use the exact Mie series scattering solution for a uniform dielectric sphere. Independent Monte-Carlo realizations of the random media are created by randomly introducing smaller spheres within the fictitious boundary until the desired volume fraction is achieved. Once the aggregate T-matrix is determined, 


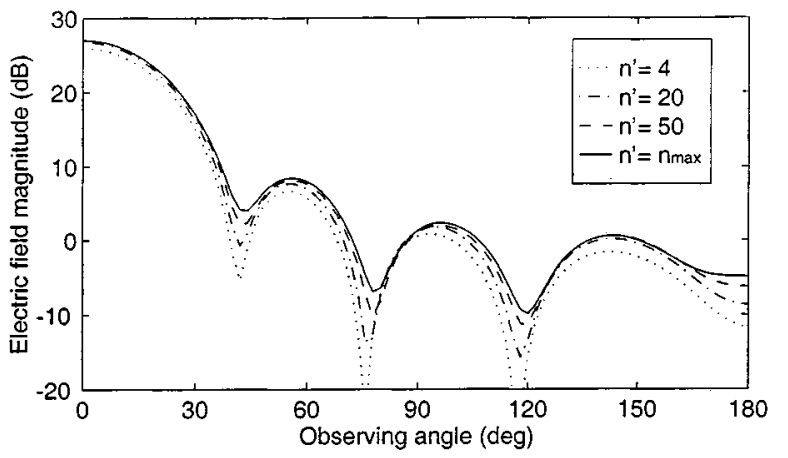

Fig. 8. Scattered field magnitude from 50 realizations of a $20 \%$ volume fraction random medium calculated using different values of $n^{\prime}$ (number of spheres added at each iteration) using the recursive aggregate T-matrix algorithm. When $n^{\prime}=n_{\max }$, RATMA is the equivalent of the direct T-matrix algorithm (in this case $n_{\max }=115$ ).

an incident field direction is chosen and the bistatic scattering pattern for both polarizations is calculated and averaged with previously calculated fields. A single realization of particle positions (and T-matrix calculation) can be used to calculate multiple bistatic scattering patterns by slightly altering the viewing geometry. Some 300 separate scattered fields were calculated for each realization in this manner. The resulting average field is then matched with the Mie series solution for a uniform dielectric sphere with the same diameter as the fictional boundary to determine the effective permittivity.

When the approach to the 3-D problem was first being designed, it was thought that the recursive aggregate T-matrix algorithm (RATMA) developed in the previous section would be an ideal tool for the analysis of effective permittivity due to its apparent computational efficiency. As was discussed, however, RATMA is limited in its ability to efficiently calculate the multiple interactions between two neighboring spheres. This limitation has the effect of increasing the scattering loss because terms in the spherical wave expansion (i.e., energy) are eliminated in the vector translation formulas. This problem is exacerbated as the volume fraction increases because multiple particle interactions also increase with volume fraction. Fig. 8 demonstrates this effect by showing the average bistatic scattered field from a $20 \%$ volume fraction medium confined to a fictitious spherical boundary with a diameter of $1.3 \lambda$ using different quantities of spheres that are added by RATMA at each iteration. The direct T-matrix solution occurs when all of the spheres are added during the first iteration (i.e., $n^{\prime}=n_{\max }$ ) and is assumed to be numerically exact. The differences seen in the scattered field patterns calculated by RATMA compared to the direct T-matrix approach, illustrated in Fig. 8, are large enough to eliminate the possibility of using RATMA for the purpose of determining effective permittivity. Basically, the apparently small errors in the bistatic pattern cause a significant error in determining the imaginary part of the effective permittivity.

The consequence of this limitation is that only the direct T-matrix algorithm can be used for solving for the scattered field from a collection of spheres. The direct T-matrix, however, is computationally limited because it requires the inversion of a large matrix whose dimension is directly proportional to the number of spheres that compose the composite scatterer.
In general, it is not feasible to accommodate a large number of spheres into the direct T-matrix algorithm; thus, an upper limit is placed on the diameter of the fictitious spherical boundary. This limit is proportional to: 1) the volume fraction of the scatterers and 2) the diameter of the subscatterers. Because of these limitations it was necessary to perform an in-depth sensitivity analysis to determine if the coherent scattered field is sufficiently sensitive to accurately determine the effective permittivity. This was done using an analytic Mie series solution for the scattering from a dielectric sphere with complex permittivity. To detect a \pm 0.01 change in the imaginary componenet of permittivity, it was determined that approximately 8000 scattered field calculations would be necessary for a bounding sphere with a diameter of $k D=4.2$. For the simulations that follow, 10000 scattered fields were averaged together to determine the mean scattered field.

\section{A. Examples For Calculation of $\epsilon_{\mathrm{eff}}$ in Three Dimensions}

For the example that is about to be developed, glass spheres $(\epsilon=6.93+i 0.1)$ with a radius of $\lambda / 10$ are used as inclusions. Glass beads are chosen for this demonstration for several reasons: 1) this material has been measured experimentally by [7] and 2) the dielectric contrast between the glass and the background (free-space) is sufficient to ensure that the beads interact strongly with the incident field. The imaginary component of the sphere permittivity serves to attenuate the scattered field, thus allowing the number of included spheres necessary to achieve large number statistics to be less than if the inclusions were lossless.

In the first set of examples, the beads are placed randomly within a fictitious spherical boundary, without overlapping, until the desired volume fraction is obtained (volume fractions up to $40 \%$ may be obtained in this manner). This method of arranging particles within a space is equivalent to the ideal fluid numerical solution given by [9] and is spherically symmetric. The average scattered field patterns obtained from 50 independent realizations and the best fit dielectric sphere $($ diameter $=1.3 \lambda$ ) solutions for $10 \%, 20 \%, 30 \%$, and $40 \%$ volume fractions were calculated. Fig. 9 gives a sample of these results for the large volume fraction case where it can be seen that there is a good fit between the dielectric sphere solution and the averaged scattered fields obtained from the Monte-Carlo simulations. A plot of the differences between the Mie scattering solution and the averaged scattered fields from the random medium as a function of both the real and imaginary components of the homogeneous sphere's permittivity is given in Fig. 10. It can be seen from this plot that there is a local minimum within the realistic range of real and imaginary permittivity for the volume fraction of $20 \%$. Similar behavior is observed at the $10 \%, 30 \%$, and $40 \%$ volume fractions studied in this section.

\section{B. Dependence on Volume Fraction and Particle Size}

The results of fitting the Mie solution for the homogeneous dielectric sphere to the coherent observed fields from the random medium sample at different volume fractions and particle sizes can be compiled into a single plot (similar to the 2-D treatment in [13]) and the results compared to theoretical methods. For the 3-D treatment discussed here, the scope of this 

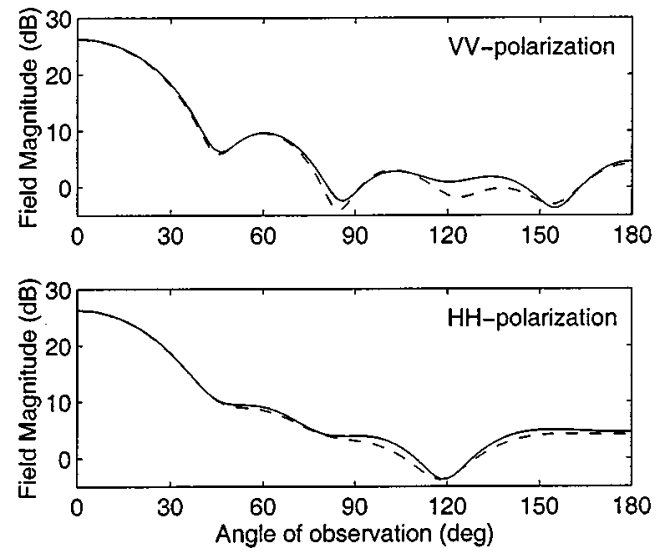

Fig. 9. Comparison between the average scattered fields for a random medium with $40 \%$ volume fraction (solid line) and the best fit Mie solution for a homogeneous dielectric sphere (dashed line) with $\epsilon_{\text {eff }}=1.90+i 0.048$.

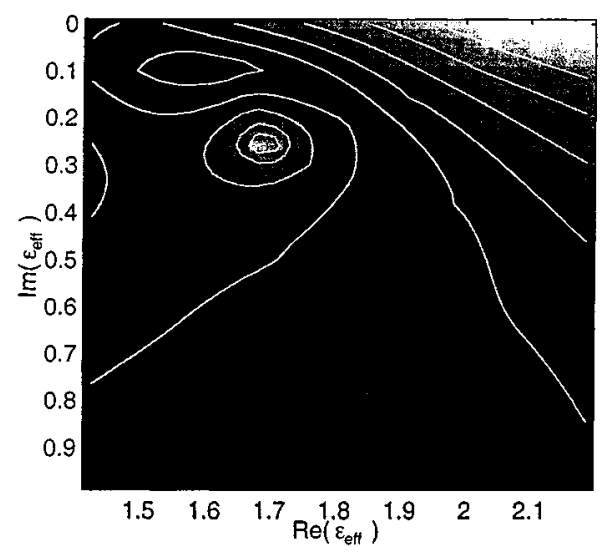

Fig. 10. Contour and gray-scale plot of the differences between the Mie solution for a dielectric sphere and the averaged scattered field obtained from the random medium as a function of real and imaginary effective permittivity. In this example, the volume fraction is $20 \%$ and the permittivity of the inclusions is $\epsilon=6.93+i 0.1$. Dark areas represent areas where the difference is the least between the two solutions.

comparison is currently limited to a narrow range of particle sizes and volume fractions due to computational considerations, the origins of which are discussed in the following section. In this context, it is possible to explore volume fractions ranging from $10 \%$ to $40 \%$ and particle sizes whose radius at $10 \mathrm{GHz}$ varies from 3 to $3.5 \mathrm{~mm}$ (i.e., diameter varies from $\lambda / 5$ to N/4.3). This range is sufficient enough to explore the effects of increased scattering losses due to larger particle sizes and the behavior of both the real and imaginary components of effective permittivity as a function of particle density.

Fundamental quantities of interest over which to compare to theoretical methods are the effective refractive index $\left(n_{\text {eff }}=\right.$ $\left.\sqrt{\epsilon_{\mathrm{eff}}}\right)$ and the normalized extinction coefficient given by

$$
\kappa_{e} / k=2 \operatorname{Im}\left(\sqrt{\epsilon_{\mathrm{eff}}}\right) .
$$

The real part of $n_{\mathrm{eff}}$ is directly proportional to the phase delay of an electromagnetic field as it passes through the random medium and $\kappa_{e} / k$ is a measure of the power lost into the incoherent field. Fig. 11 illustrates the numerically calculated results and provides a comparison to the theoretical models of the quasi-crystalline approximation with coherent potential
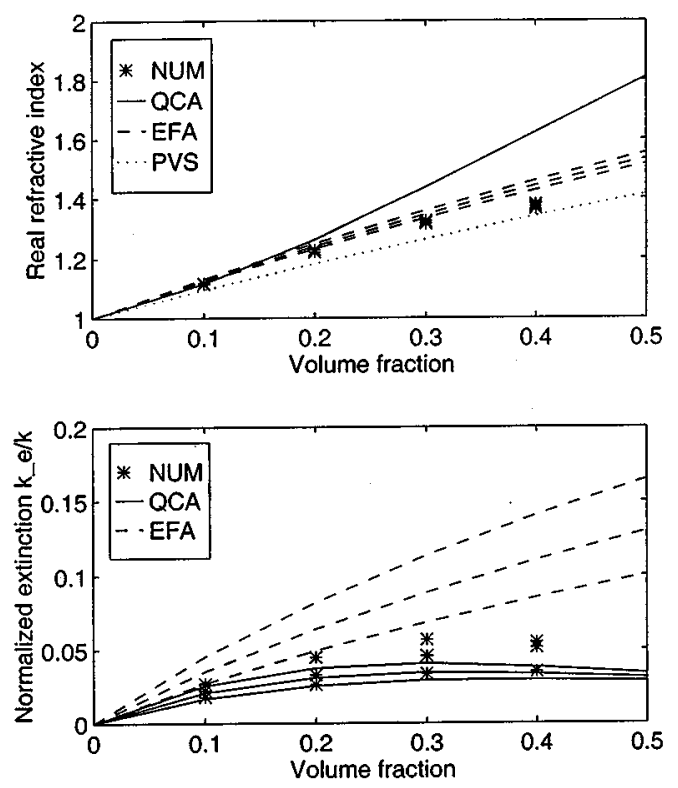

Fig. 11. Real refractive index and normalized extinction coefficient comparison between the numerical method (symbols) and the theoretical techniques of quasi-crystalline approximation with coherent potential (solid lines) and the effective field approximation (dashed lines) for three particle radii ranging from $k a=0.63$ to 0.73 . Normalized extinction (shown in the lower plot) consistently increases with particle size for all three methods shown. In this series of numerical experiments, $P_{\max }=17$.

(QCA, solid lines), the effective field approximation (EFA, dashed lines), and the Polder-Van Santen mixing formula (PVS, dotted line).

The first plot of Fig. 11 compares the real component of $n_{\text {eff }}$, which is not expected to vary strongly as a function of particle size. This is a characteristic that is common between the theoretical and numerical method results. The three separate dashed lines in this plot indicate theoretical results from the effective field approximation for the three different particle sizes with the effect of increasing particle size causing an increase in real refractive index. The numerical results obtained fall closely between the effective field approximation and the Polder-Van Santen mixing formula using a background dielectric of free-space. The numerical calculations seem to indicate that the behavior of the fields near the inclusions at these volume fractions experience the full dielectric contrast between the inclusions and the host material rather than the dielectric contrast between the included material and the effective permittivity of the random medium. At some point not modeled here it is expected that this behavior should change as volume fraction increases and the medium becomes one dominated by inclusions rather than the spaces between inclusions. In this case, the background dielectric would no longer be similar to that of free-space (as the numerical technique in this example would indicate) rather it would be closer to that of the effective permittivity $\epsilon_{\text {eff. }}$. The manner in which particles are arranged within the volume may have an effect on this behavior as in this example, where the method of particle arrangement maximizes the average distance between inclusions.

The second plot of Fig. 11 displays the normalized extinction coefficient given by (35), which reflects the power lost to 
the incoherent field. The numerical method is compared with the theoretical methods of the quasicrystalline approximation with coherent potential and the effective field approximation (the supposed upper limit). Each of the method results is given in sets of three for each of the three particle sizes used (radius, $a=3,3.25,3.5 \mathrm{~mm}$ at $10 \mathrm{GHz}$ or $k a=0.63,0.68$, and 0.73 ), with the larger particle sizes having the effect of increasing $\kappa_{e} / k$ for all three methods. It is evident from this illustration that at $10 \%$ volume fraction, that the numerical method and QCA-CP agree closely for the three particle sizes, while as particle density increases, the two methods diverge with QCA-CP predicting lower scattering losses than the numerical method. This likely reflects the natural limitation of QCA-CP to address particle interactions that occur in groups larger than two (i.e., QCA models multiple interactions between pairs of particles and excludes the effect three or greater particle interactions). This change occurs most predominantly for volume fractions of $25 \%$ and greater and is more pronounced for larger particle sizes, an unsurprising result given that the general accepted limitation for QCA is for $k a$ less than 0.2 or 0.3 .

\section{Limitations of the 3-D Technique}

In the previous subsection, the coherent numerical method presented in this chapter was used to compare with similar results obtained using theoretical methods for determining the extinction coefficient. In this manner, the numerical technique was used to explore the limitations of the other methods. The 3-D determination of effective permittivity, however, has its own set of limitations that are imposed due to computational concerns. Fig. 12 attempts to graphically display these limits as they have been explored thus far and to illustrate those points that have been demonstrated in the previous subsection (indicated by x's).

The lower limit of the bounded region is labeled as $N_{\max }$ in reference to the fact that the direct T-matrix cannot simultaneously solve for more than 150 Rayleigh-sized spheres on a convex MPP-1000 parallel computer with approximately 500 $\mathrm{Mb}$ of random access memory. Using less spheres would require the use of a smaller bounding sphere, thus decreasing the sensitivity of the coherent method to the imaginary component of effective permittivity.

The volume fraction limit, labeled "Packing Method" refers to an upper limit on the volume fraction that can be obtained using the random introduction of spheres into an empty space (i.e., simulation of the Percus-Yevick pair distribution function for an ideal fluid). While other packing algorithms may be used to surpass this limit, the isotropy of the medium can no longer be guaranteed [12] and, in turn, it may no longer be acceptable to alter the geometry of the incident field and the plane of observation to obtain a sufficient number of independent samples. This would have the effect of increasing the number of independent realizations to an impractical number and, thus, the average scattered field may not be representative of a homogeneous dielectric.

The upper limit which dictates the maximum radius allowed for the inclusions is imposed because larger inclusions require more terms in the spherical wave expansion $\left(K_{\max }\right)$ in addition to the fact that a large boundary dimension may be required to enclose a sufficient number of inclusions to accurately repre-

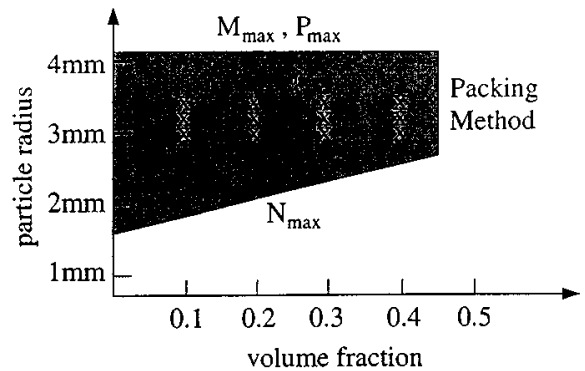

Fig. 12. Illustration of the volume fraction and particle radius limits for the determination of effective permittivity using the coherent method in three dimensions. $X$ 's illustrate those points that have been calculated in the previous subsections (i.e., radius $=3 \mathrm{~mm}$ to $3.5 \mathrm{~mm}$ and volume fraction ranging from $10 \%$ to $40 \%)$. The observing frequency is $10 \mathrm{GHz}(\lambda=3 \mathrm{~cm})$.

sent the random medium. This large boundary is limited by the maximum number of spherical waves $\left(P_{\max }\right)$ computationally allowed to represent the aggregated T-matrix (for our facilities, this was approximately 20).

\section{CONCLUSIONS AND RECOMMENDATIONS}

In this paper, a 3-D coherent field technique for determining effective permittivity for random media was developed. We began by illustrating the success of this method for addressing a 2-D problem and then discussed in-depth the 3-D T-matrix numerical technique that was to be applied for solving Maxwell's equations. One possible version of this RATMA technique was demonstrated to be insufficient for addressing this problem.

For the numerical technique itself, between 30 and 50 independent realizations of the random medium were required to converge on a representative coherent field. This coherent field can be used to determine effective permittivity. A complete example was illustrated using a particle diameter of $\lambda / 5$ (radius, $a=3 \mathrm{~mm}$ at $10 \mathrm{GHz}$ ) for volume fractions ranging from 10 to $40 \%$ where it was shown that the average scattered field obtained from the Monte-Carlo simulations agreed well with a Mie series solution for a homogeneous dielectric sphere. Additionally, the differences between the Mie series solution and the averaged scattered field were shown to have an isolated local minimum within the physical range of $\epsilon_{\mathrm{eff}}$ for a representative volume fraction of $20 \%$. This example was then expanded to include two other particle diameters of $\lambda / 4.6(a=3.25 \mathrm{~mm})$ and $\lambda / 4.3(a=3.5 \mathrm{~mm})$ where a comparison was made between the numerical method and the theoretical methods of the quasi-crystalline approximation with coherent potential and the effective field approximation. It was shown that the numerical method predicted a lower real component of the index of refraction than these theoretical models and predicted an extinction coefficient that agreed well with QCA-CP at low-volume fractions and the smaller of the particle diameters. At higher volume fractions and large particle diameters, the numerical technique indicates larger scattering losses, with a higher order of particle interaction than QCA-CP theoretically accounts for (i.e., two-particle interactions).

A discussion was then provided regarding the current limitations of the coherent technique in three dimensions. These limitations are imposed by the computational technique that has been implemented (direct T-matrix approach) and 
may be broadened as the technique is used to explore a wider range of problems. It is recommended that the technique is expanded/verified in the future by: 1) exploring a variety of different boundary shapes and sizes to maximize the sensitivity of the coherent field technique to the imaginary component of effective permittivity (the dielectric slab used in two dimensions seems to be one such possibility); 2) implementing and comparing alternative numerical techniques for solving scattered fields; and 3) using the results that have been obtained in this paper to explore theoretical/numerical methods of reducing the computational burden that a full Monte-Carlo simulation that the problem entails.

\section{ACKNOWLEDGMENT}

The authors would like to thank the Advanced Laboratory for Parallel High-Performance Applications Group at the Jet Propulsion Laboratory (JPL), Pasadena, CA, for technical assistance and S. Lagault, University of Michigan, Ann Arbor, for his useful discussions.

\section{REFERENCES}

[1] M. Abramowitz and I. Stegun, Handbook of Mathematical Functions. New York: Dover, 1965, p. 1046.

[2] C. Bohren and D. Huffman, Absorption and Scattering of Light by Small Particles. New York: Wiley, 1983, p. 519.

[3] W. C. Chew, Waves and Fields in Inhomogeneous Media. New York: IEEE Press, 1990, p. 608

[4] —, "Recurrence relations for three-dimensional scalar addition theorem," J. Electromagn. Waves Applicat., vol. 6, no. 2, pp. 133-142, 1992.

[5] W. C. Chew, Y. M. Wang, and L. Gurel, "Recursive algorithm for wavescattering solutions using windowed addition theorem," J. Electromagn. Waves Applicat., vol. 6, no. 11, pp. 1537-1560, 1992.

[6] W. C. Chew and C. C. Lu, "The resurive aggregate interaction matrix algorithm for multiple scatterers," IEEE Trans. Antennas Propagat., vol. 43, pp. 1483-1486, Feb. 1995.

[7] A. Nashashibi and K. Sarabandi, "A technique for measuring the effective propagation constant of dense random media," in IEEE AP-S Int. Symp., Newport Beach, CA, June 1995, pp. 748-751.

[8] J. Percus and G. Yevick, "Analysis of classical statistical mechanics by means of collective coordinates," Phys. Rev., vol. 110, pp. 1-113, 1958.

[9] B. Peterson and S. Storm, "T-matrix for electromagnetic scattering from an arbitrary number of scatterers and representations of E(3)," Phys. Rev. $D$, vol. 8, no. 10, pp. 3661-3678, 1973.

[10] D. Polder and J. H. VanSanten, "The effective permeability of mixtures of solids," Physica, vol. 12, no. 5, pp. 1257-1271, 1946.

[11] K. Sarabandi and P. R. Siqueira, "Numerical scattering analysis for twodimensional dense random media," in IEEE Antennas Propagat. Conf. Proc., Seattle, WA, May 1994, pp. 858-867.

[12] _ _ "Numerical scattering analysis for two-dimensional dense random media: Characterization of effective permittivity," IEEE Trans. Antennas Propagat., vol. 45, pp. 858-867, May 1997.

[13] P. R. Siqueira, K. Sarabandi, and F. T. Ulaby, "Numerical simulation of scatterer positions in a very dense media with an application to the two-dimensional born approximation," Radio Sci., vol. 30, no. 5, pp. 1325-1339, Sept./Oct. 1995.

[14] P. Siqueira and K. Sarabandi, "Method of moments evaluation of the two-dimensional quasicrystalline approximation," IEEE Trans. Antennas Propagat., vol. 44, pp. 1067-1077, Aug. 1996.
[15] L. Tsang, J. Kong, and R. Shin, Theory of Microwave Remote Sensing. New York: Wiley, 1985.

[16] L. Tsang, C. E. Mandt, and K. H. Ding, "Monte Carlo simulations of the extinction rate of dense media with randomly distributed dielectric spheres based on solution of Maxwell's equations," Opt. Lett., vol. 17, no. 5, pp. 314-316, 1992.

[17] Y. M. Wang and W. C. Chew, "A recursive T-matrix approach for the solution of electromagnetic scattering by many spheres," IEEE Trans. Antennas Propagat., vol. 14, pp. 1633-1639, Dec. 1993.

[18] P. C. Waterman, "Matrix formulation of electromagnetic scattering," in IEEE Proc., vol. 53, Aug. 1965, pp. 805-812.

[19] L. M. Zurk, L. Tsang, K. H. Ding, and D. P. Winebrenner, "Monte Carlo simulations of the extinction rate of densely packed spheres with clustered and nonclustered geometries," J. Opt. Soc. Amer. A, vol. 12, pp. 1772-1781, 1995.

[20] L. M. Zurk, L. Tsang, and D. P. Winebrenner, "Scattering properties of dense media from Monte Carlo simulations with the application to active remote sensing of snow," Radio Sci., vol. 31, pp. 803-819, 1996.

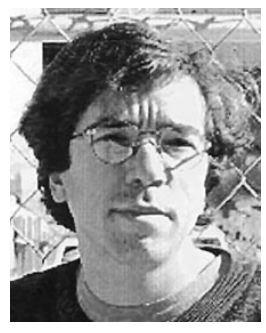

Paul Siqueira (S'92-M'96) was born in Skokie, IL, in 1966. He received the B.S. and M.S. degrees in electrical engineering from Iowa State University, Ames, in 1987 and 1990, respectively, and the Ph.D. degree in electrical engineering from the Radiation Laboratory, University of Michigan, Ann Arbor, in 1996

From 1990 to 1991, he worked at the Astronomy and Molecular Biology Departments, University of Chicago, IL. He is currently working as a Staff Engineer performing system engineering and radar data analysis for the Radar Science and Engineering Section, Jet Propulsion Laboratory, Pasadena, CA. His current research interests are in the modeling and characterization of polarimetric/interferometric measurements of random media.

Kamal Sarabandi (S'87-M'90-SM'92) received the B.S. degree in electrical engineering from Sharif University of Technology, Tehran, Iran, in 1980, the M.S.E. (electrical engineering) degree from the University of Michigan, in 1986, and the M.S. (mathematics) and Ph.D. (electrical engineering) degrees from the University of Michigan, Ann Arbor, in 1989.

From 1980 to 1984, he worked as a Microwave Engineer in the Telecommunication Research Center. He is presently an Associate Professor in the Department of Electrical Engineering and Computer Science, University of Michigan. He has 18 years of experience with microwave sensors and radar systems. In the past eight years he has served as the Principal Investigator and Co-Investigator on many projects sponsored by National Aeronautics and Space Administration (NASA), Jet Propulsion Laboratory (JPL), Army Research Office (ARO), Office of Naval Research (ONR), Army Research Labs (ARL), and General Motors (GM) all related in one way or another to microwave and millimeter-wave radar remote sensing. He has published many book chapters and more than 80 papers in refereed journals on electromagnetic scattering, random media modeling, microwave measurement techniques, radar calibration, application of neural networks in inverse scattering problems, and microwave sensors. He has also had more than 140 papers and invited presentations in national and international conferences and symposia on similar subjects.

Dr. Sarabandi is listed in American Men and Women of Science and Who's Who in Electromagnetics. He is a member of the IEEE Geoscience and Remote Sensing ADCOM since January of 1998 and served as the Chairman of Geoscience and Remote Sensing Society Southeastern Michigan chapter from 1992 to 1998. He is also a member of Commission F of URSI and the Electromagnetic Academy. He was a recipient of a 1996 Technical Excellence Award, the 1997 Henry Russel Award from the Regent of The University of Michigan, and the 1999 GAAC Distinguished Lecturer Award from the German Federal Ministry for Education, Science, and Technology. 